\title{
Pathologically Benign Lymph Nodes Can Mimic Malignancy on Imaging in Patients With Angiomatoid Fibrous Histiocytoma
}

\author{
Gary A. Ulaner MD, PhD, John H. Healey MD, Edward A. Athanasian MD
}

Received: 12 January 2017/ Accepted: 16 May 2017/Published online: 23 May 2017

(C) The Association of Bone and Joint Surgeons (B) 2017

\begin{abstract}
Background Angiomatoid fibrous histiocytoma (AFH) is a rare soft tissue neoplasm, and its rarity makes studying it difficult. We found that several of our patients with AFH presented with radiologically suspicious local lymph nodes that were sampled because of their imaging characteristics, but the nodes proved to be benign on pathologic evaluation. Although the frequency of this finding is unknown, it seems important for orthopaedic oncologists who care for patients with AFH to know whether suspicious-appearing
\end{abstract}

The institution of the authors (GAU, JHH, EAA) has received funding from the National Institutes of Health/National Cancer Institute Cancer Center Support Grant P30 CA008748.

All ICMJE Conflict of Interest Forms for authors and Clinical Orthopaedics and Related Research ${ }^{\mathbb{R}}$ editors and board members are on file with the publication and can be viewed on request. Each author certifies that his or her institution approved or waived approval for the human protocol for this investigation and that all investigations were conducted in conformity with ethical principles of research

This work was performed at Memorial Sloan Kettering Cancer Center, New York, NY, USA.

G. A. Ulaner ( $\square)$

Department of Radiology, Memorial Sloan Kettering Cancer Center, 1275 York Avenue, Box 77, New York, NY 10065, USA e-mail: ulanerg@mskcc.org

G. A. Ulaner

Department of Radiology, Weill Cornell Medical College, New York, NY, USA

J. H. Healey, E. A. Athanasian

Department of Surgery, Memorial Sloan Kettering Cancer

Center, New York, NY, USA

J. H. Healey, E. A. Athanasian

Department of Surgery, Weill Cornell Medical College, New York, NY, USA associated nodes in these patients warrant aggressive management.

Questions/purposes (1) How often do patients with newly diagnosed AFH present with radiologically suspicious lymph nodes? (2) How often are the radiologically suspicious nodes malignant on pathologic evaluation?

Methods In this retrospective, Health Insurance Portability and Accountability Act-compliant study, we used a hospital database to identify all 54 patients treated at our hospital for AFH between 1993 and 2016. This study was performed with institutional review board waiver. All of the patients were considered potentially eligible for analysis. Of the patients, 19 (35\%) had pretherapy imaging; during the period in question, pretherapy imaging generally was obtained when there was uncertainty regarding extent of disease. All patients who had imaging underwent MRI, and four also had fluorodeoxyglucose positron emission tomography (FDG PET/CT). Imaging reports were reviewed to identify which patients had nodes that were called suspicious in the reports. All patients with nodes described as suspicious on imaging underwent subsequent pathologic analysis for the presence or absence of metastatic AFH cells in the node.

Results Seven of 19 patients with pretherapy imaging had local lymph nodes called suspicious for nodal metastases. Pathologic analysis of these nodes showed they were malignant in only one patient, whereas six patients had nodes that were histologically benign despite suspicious imaging findings. Benign nodes measured as much as $3.2 \times 1.8 \mathrm{~cm}$ on MRI and showed maximum standardized uptake values up to 10.9 on FDG PET/CT.

Conclusions Patients with newly diagnosed AFH present with benign lymph nodes that are mistaken for malignancy on imaging. Orthopaedic surgeons and radiologists should be aware of this finding in patients with AFH. Less- 
invasive management of suspicious nodes, such as imageguided biopsy, may be preferable to nodal resections, as this will help decrease the aggressiveness of surgery for patients with newly diagnosed AFH.

Level of Evidence Level IV, diagnostic study.

\section{Introduction}

Angiomatoid fibrous histiocytoma ( $\mathrm{AFH})$ is a rare soft tissue neoplasm of unclear origin [14]. It is most commonly found superficially in the extremities of patients younger than 30 years [3, 5], although it has been found in other sites including the soft tissues of the neck and trunk, brain, lungs, peritoneum, retroperitoneum, gynecologic tract, and bone, and at ages ranging from infancy to the elderly [2, 14]. AFH was first reported as a subtype of malignant fibrous histiocytoma [4], but has since been reclassified as a distinct pathologic process [3]. Most AFHs have an indolent course with local recurrences in only $15 \%$ of cases and even lower rates of metastases. AFH has three characteristic chromosomal translocations $[1,11]$, which are important in diagnosis, because histopathologic findings of AFH may be confused with several other benign and malignant lesions [7, 12-14].

The management of AFH is considered to be wide local excision and followup for recurrence; however, with unresectable or metastatic disease, adjuvant chemotherapy and/ or radiation therapy may be used [14]. Thus, appropriate staging of nodal and distant metastases affects patient management. Local staging often is assisted by MRI [10], and when appropriate, staging may be augmented by fluorodeoxyglucose positron emission tomography (FDG PET/ CT) [9]. At our institution, we noted a subset of patients with newly diagnosed AFH who presented with suspiciously enlarged or FDG-avid lymph nodes on MRI and (in those who had this modality performed) FDG PET/CT, but in whom histologic evaluation revealed the nodes to be benign. Although the frequency of this finding is unknown, it seems important for orthopaedic oncologists who care for patients with AFH to know whether suspicious-appearing associated nodes in these patients warrant aggressive management.

We therefore performed a retrospective study to determine: (1) how often patients with newly diagnosed $\mathrm{AFH}$ present with radiologically suspicious lymph nodes, and (2) how often the radiologically suspicious nodes are malignant on pathologic evaluation?

\section{Patients and Methods}

This retrospective, single-institution study was performed in compliance with the Health Insurance Portability and
Accountability Act and approved by the institutional review board. As this study was retrospective, without effect on patient management, the requirement to obtain informed consent was waived by the board. The Memorial Sloan Kettering Cancer Center health information system was searched for patients with a diagnosis of AFH from 1993 to 2016.

Fifty-four patients with AFH were identified (28 male and 26 female patients), with an age range of 2 to 79 years (median, 22 years) (Table 1). All of these patients were considered potentially eligible for analysis. The health information system then was reviewed to determine which of these patients underwent cross-sectional staging imaging (MRI, CT, and/or FDG PET/CT) at initial diagnosis, before resection or therapy. Nineteen of 54 (35\%) patients underwent pretherapy imaging; all 19 (100\%) had MRI and four of $19(21 \%)$ also had FDG PET/CT. In general, MRI was used when the extent of local disease was uncertain, and FDG PET/CT was used when there was concern for unsuspected sites of disease. MRI reports were reviewed to document whether nodes were called suspicious for nodal metastases and to document lymph node sizes. No patients underwent $\mathrm{CT}$ at initial diagnosis. FDG PET/CT reports were reviewed to document whether nodes were called suspicious for nodal metastases and for maximum standardized uptake value (SUVmax).

In total, seven of 19 patients $(37 \%)$ with pretherapy imaging had imaging findings suspicious for nodal metastases. All seven patients with suspicious nodes on pretherapy imaging had subsequent pathologic evaluation of the nodes by biopsy $(n=$ three) or nodal resection $(\mathrm{n}=$ four). When performed as nodal resections they were

Table 1. Characteristics of patients with AFH $(n=54)$

\begin{tabular}{ll}
\hline Patient characteristic & Result \\
\hline Age (median, range) & 22 years (2-79 years) \\
Gender & \\
Male & $28(52 \%)$ \\
Female & $26(48 \%)$ \\
Primary AFH site & \\
Soft tissues of the: & \\
Head/neck & $8(15 \%)$ \\
Trunk & $14(26 \%)$ \\
Extremities & $26(48 \%)$ \\
Other locations & \\
Bone & $3(5 \%)$ \\
Brain & $1(2 \%)$ \\
Pleura & $1(2 \%)$ \\
Peritoneum & $1(2 \%)$ \\
\hline AFH
\end{tabular}

$\mathrm{AFH}=$ angiomatoid fibrous histiocytoma. 
Table 2. Patients with AFH undergoing pretherapy imaging $(\mathrm{n}=19)$

\begin{tabular}{|c|c|c|c|c|c|c|c|c|}
\hline $\begin{array}{l}\text { Patient } \\
\text { number }\end{array}$ & $\begin{array}{l}\text { Age } \\
\text { (years) }\end{array}$ & Gender & $\begin{array}{l}\text { Primary AFH } \\
\text { site }\end{array}$ & $\begin{array}{l}\text { Year of primary } \\
\text { diagnosis }\end{array}$ & $\begin{array}{l}\text { Suspicious nodes on } \\
\text { imaging? }\end{array}$ & $\begin{array}{l}\text { Size on MRI } \\
(\mathrm{mm})\end{array}$ & $\begin{array}{l}\text { SUV on } \\
\text { PET/CT }\end{array}$ & $\begin{array}{l}\text { Pathology of } \\
\text { nodes }\end{array}$ \\
\hline 1 & 5 & $\mathrm{~F}$ & Right gluteal & 2007 & No & & & \\
\hline 2 & 6 & $\mathrm{~F}$ & Left thigh & 2007 & No & & & \\
\hline 3 & 7 & M & Right forearm & 2016 & Yes-antecubital & $8 \times 8$ & 3.0 & Benign \\
\hline 4 & 9 & $\mathrm{~F}$ & Left arm & 2005 & No & & & \\
\hline 5 & 10 & $\mathrm{~F}$ & $\begin{array}{l}\text { Right } \\
\text { shoulder }\end{array}$ & 2001 & No & & & \\
\hline 6 & 11 & M & Left arm & 1995 & Yes-axillary & $12 \times 10$ & & Benign \\
\hline 7 & 11 & $\mathrm{~F}$ & Right groin & 2004 & Yes-inguinal & $16 \times 12$ & & Benign \\
\hline 8 & 13 & M & Left axilla & 2014 & Yes-axillary & $29 \times 10$ & & Malignant \\
\hline 9 & 18 & M & Left face & 2011 & Yes-neck & $16 \times 14$ & 3.9 & Benign \\
\hline 10 & 21 & M & Right arm & 2011 & No & & & \\
\hline 11 & 22 & $\mathrm{~F}$ & Right thigh & 2003 & Yes-inguinal, iliac & $32 \times 18$ & 10.9 & Benign \\
\hline 12 & 27 & $\mathrm{~F}$ & $\begin{array}{l}\text { Right chest } \\
\text { wall }\end{array}$ & 2006 & No & & & \\
\hline 13 & 28 & $\mathrm{~F}$ & Right groin & 2000 & Yes-inguinal & $16 \times 10$ & & Benign \\
\hline 14 & 33 & M & Neck & 2009 & No & & & \\
\hline 15 & 36 & $\mathrm{~F}$ & Right thigh & 2008 & No & & & \\
\hline 16 & 39 & $\mathrm{~F}$ & $\begin{array}{l}\text { Right chest } \\
\text { wall }\end{array}$ & 2016 & No & & & \\
\hline 17 & 41 & $\mathrm{~F}$ & Left foot & 2016 & No & & & \\
\hline 18 & 55 & $\mathrm{~F}$ & Left thigh & 2011 & No & & & \\
\hline 19 & 78 & $\mathrm{~F}$ & Right knee & 2004 & No & & & \\
\hline
\end{tabular}

$\mathrm{AFH}=$ angiomatoid fibrous histiocytoma; $\mathrm{SUV}=$ standardized uptake value; $\mathrm{PET}=$ positron emission tomography.

all performed separately and not in continuity with the primary mass. Nodes were deemed metastatic if histologic evaluation showed any AFH cells.

\section{Results}

Seven of the 19 patients with pretherapy imaging had imaging findings suspicious for nodal metastases. All seven had suspicious lymph nodes noted on MRI in locoregional nodal basins. Nodal lesions measured up to $32 \times 18 \mathrm{~mm}$ (Table 2). Three patients had focally FDG-avid nodes that were considered suspicious for nodal metastases. Nodal lesions had FDG avidity of up to a SUVmax of 10.9 (Table 2).

AFH metastasis was found in the nodes of only one of the seven patients who had lymph-node sampling based on suspicious imaging findings. At last followup, approximately 1 year after diagnosis, this patient was alive with stable disease and no additional detected metastases. The remaining six patients had benign nodes on pathologic evaluation despite suspicious imaging findings. For example, one patient had local nodes measuring up to $32 \times 18 \mathrm{~mm}$ and FDG SUVmax of 10.9 on FDG PET/CT, which were considered suspicious on imaging, yet nodal pathologic evaluation was benign (Fig. 1). Another patient presented with an enlarged and FDG-avid right antecubital node, which was considered suspicious on imaging, however pathologic evaluation was benign (Fig. 2).

In a search of our Hospital Information System on all 54 patients with AFH, four had documented local recurrences and none had documentation of distant metastases. None of these four patients were among the 19 who had pretherapy imaging.

\section{Discussion}

It is important for orthopaedic oncologists who care for patients with AFH to know if associated nodes which are suspicious on imaging warrant aggressive management. We noted that several patients with AFH presented with radiologically suspicious local lymph nodes that were sampled because of their imaging characteristics, but the nodes were benign on pathologic evaluation. We decided to evaluate how common this phenomenon is. In this retrospective study, we found that patients with newly diagnosed AFH, a rare neoplasm of unclear origin, often 

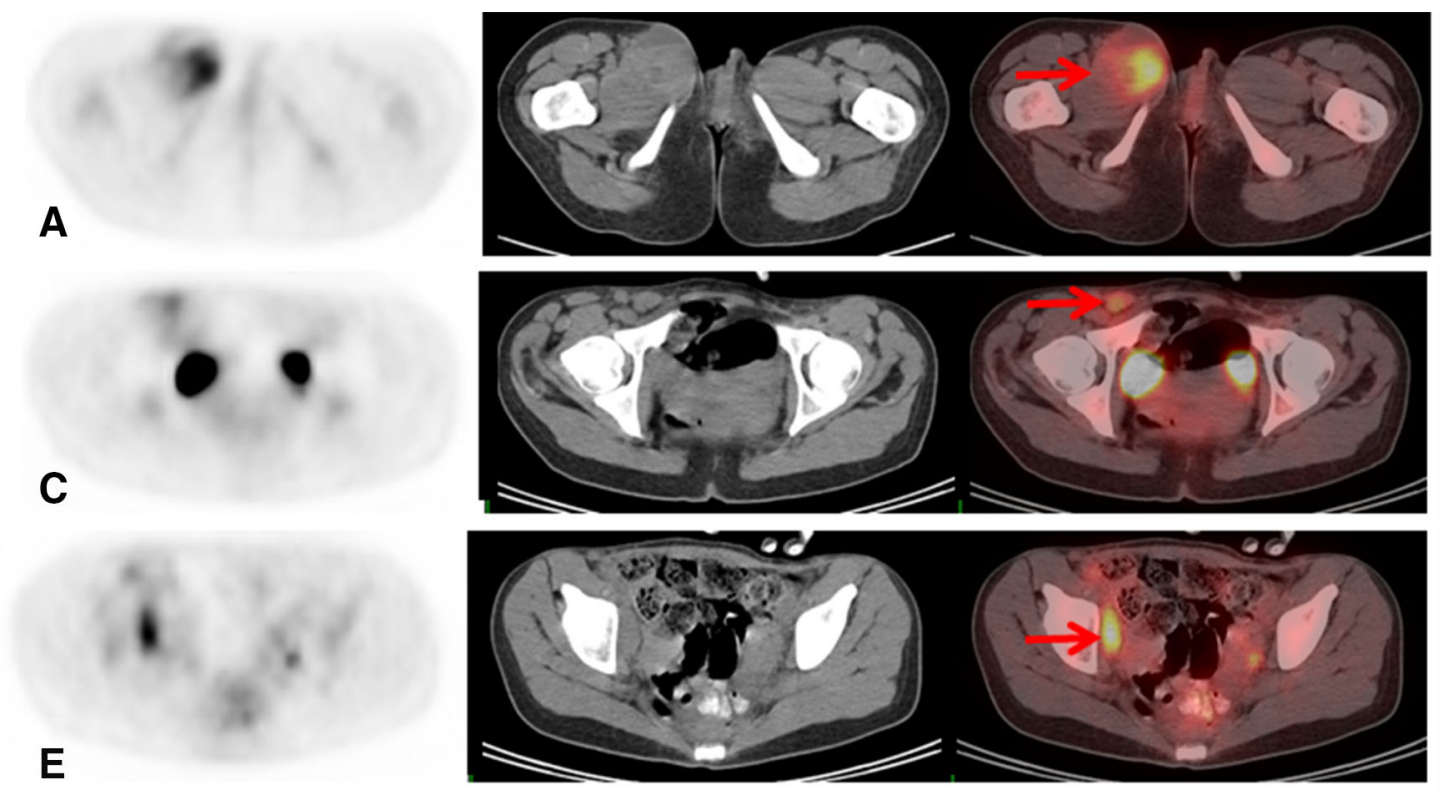
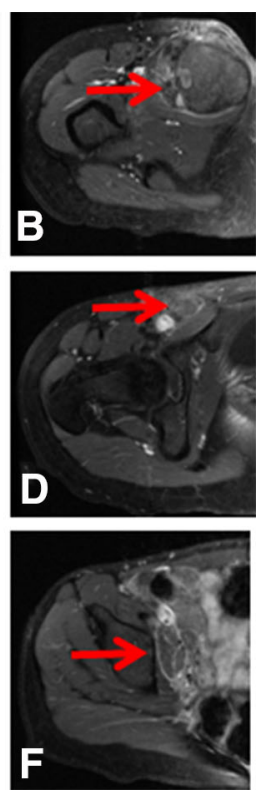

Fig. 1A-F Lymph nodes in a 22-year-old woman with primary right thigh AFH were suspicious on imaging, but benign on pathologic evaluation. (A) Axial PET, CT, and fused PET/CT images from a PET/CT study show the primary right thigh soft tissue mass (arrow on fused image). (B) An axial T1 gadolinium-enhanced MR image also shows the primary right thigh soft tissue mass (arrow). (C) Axial PET, $\mathrm{CT}$, and fused PET/CT images show an FDG-avid (SUV 2.7) right inguinal node, suspicious for nodal malignancy (arrow on fused image). (D) An axial T1 gadolinium-enhanced MR image shows the enlarged $(22 \times 14 \mathrm{~mm})$ suspicious right inguinal node (arrow). $(\mathbf{E})$ Axial PET, CT, and fused PET/CT images show an FDG-avid (SUV 10.9) right external iliac node, suspicious for nodal malignancy (arrow on fused image). (F) The axial T1 gadolinium-enhanced MR image shows the enlarged $(32 \times 18 \mathrm{~mm})$ suspicious right external iliac node (arrow). Pathologic evaluation of specimen from the surgical resection showed the malignant right thigh $\mathrm{AFH}$, but the lymph nodes were benign "with siderosis. No tumor seen."

considered suspicious by a knowledgeable radiologist. The methodology of diagnosing AFH on pathologic evaluation has changed during the period that these patients were treated, with the detection of gene fusions now playing a major role. Thus, some patients were diagnosed with AFH by evaluation of gene fusions, and others were not. We used the original pathologic diagnosis of AFH to include patients in this study. We did not readjudicate the slides. While detection of gene fusions has added to the diagnosis of $\mathrm{AFH}$, the morphologic characteristics considered characteristic of $\mathrm{AFH}$ have remained stable with time, the diagnoses were made by subspecialized musculoskeletal pathologists, and the diagnosis of AFH in these patients is appropriate. Owing to the retrospective design of this study, followup for local recurrence or metastases in these patients is based on available data in our hospital information system and thus may be incomplete.

While nodal metastases are not the most-common site of metastasis from soft tissue neoplasms, a large prospective database documents the occurrence of nodal metastases in these patients [6]. The presence of nodal metastases probably results in poorer prognosis [6]. Smaller studies have documented the use of sentinel lymph node biopsy [8] and image-guided biopsy [15] to detect nodal metastases in patients with soft tissue neoplasms. The latter study 


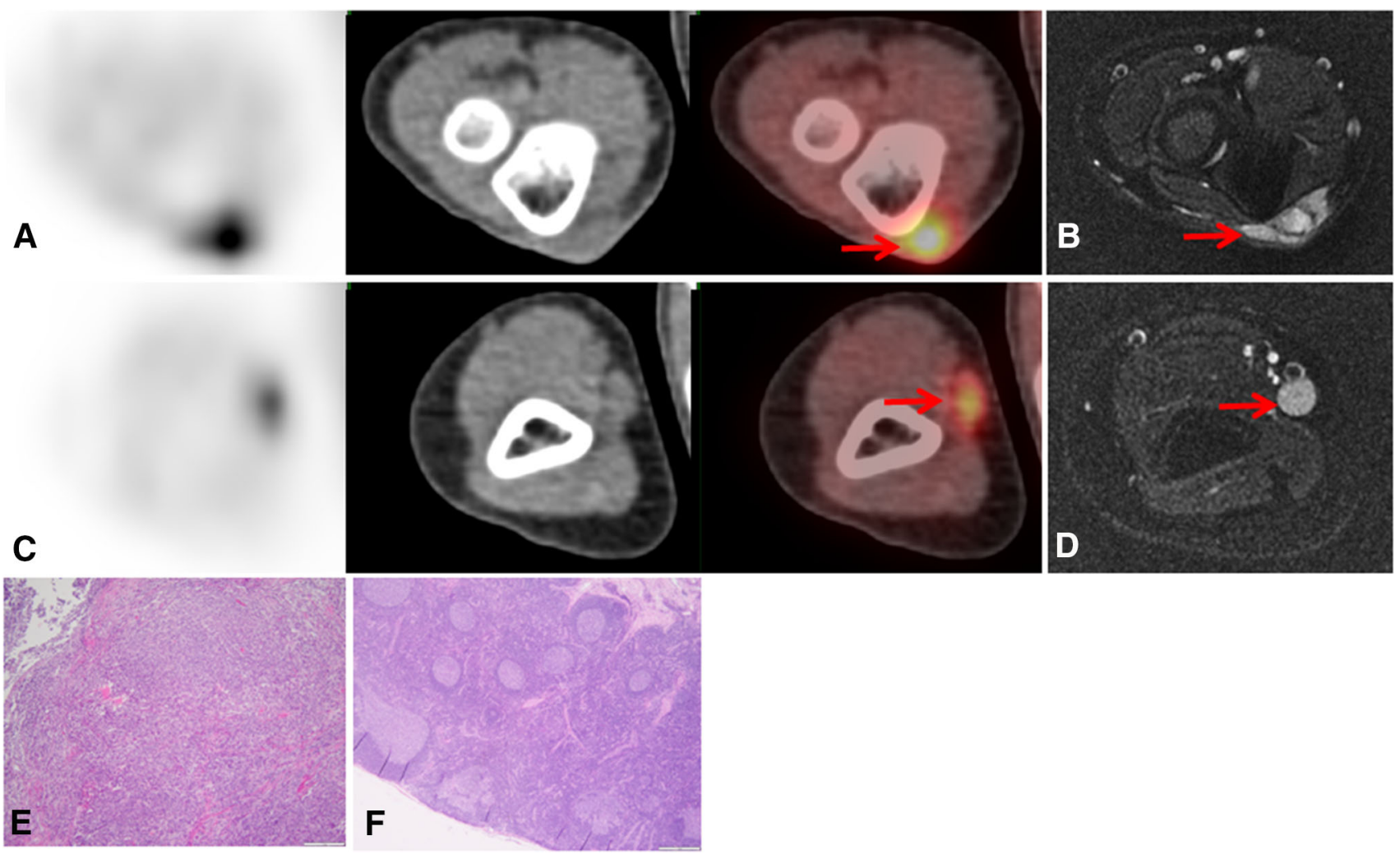

Fig. 2A-H A lymph node in a 7-year-old boy with primary right forearm AFH was suspicious on imaging, but benign on pathologic evaluation. (A) His axial PET, CT, and fused PET/CT images show the primary right forearm soft tissue mass (arrow on fused image). (B) The axial T1 gadolinium-enhanced MR image also shows the primary right forearm soft tissue mass (arrow). (C) Axial PET, CT, and fused PET/CT images show an FDG-avid (SUV 3.0) right antecubital node, suspicious for nodal malignancy (arrow on fused image). (D) An axial T1 gadolinium-enhanced MR image shows the rounded $(8 \times 8 \mathrm{~mm})$

showed suspicious nodes which were first identified on FDG PET/CT and subsequently biopsy proven to be nodal metastases in a patient with a primary soft tissue Ewing sarcoma [15]. Thus, suspicious nodes on imaging may lead to the diagnosis of nodal metastases in patients with soft tissue malignancies. To our knowledge, this is the first published report of patients with AFH showing a high rate of suspicious nodes on imaging that are not malignant. Only one in seven patients with newly diagnosed AFH with suspicious nodes on imaging showed nodal metastases on pathologic evaluation. In patients with $\mathrm{AFH}$, suspicious nodes on imaging are often benign, and may be evaluated by less-invasive management, such as image-guided biopsy rather than nodal resection, if this will help decrease the aggressiveness of surgery with curative intent.

The etiology of the benign nodal enlargement and FDG avidity is unclear. Pathologically, most AFHs have a rim of lymphoid tissue that has been believed to represent an effaced lymph node and thus AFH may arise from nodal suspicious right antecubital node (arrow). (E) Pathologic evaluation of the right forearm soft tissue mass showed spindle tumor cells and was diagnostic of AFH (Stain, hematoxylin \& eosin; original magnification, $\times 40$ ). (F) Pathologic evaluation of the antecubital node which was suspicious on imaging showed a reactive lymph node with large follicles and germinal centers, but no evidence of malignancy (Stain, hematoxylin \& eosin; original magnification, $\times 40)$.

tissue [14]. Lymph nodes usually are not enlarged or FDGavid unless involved with malignancy or inflammation, yet the potential lymphoid origin of this neoplasm provides a possible explanation for changes in local nodes. Although AFH is relatively indolent compared with many soft tissue neoplasms, recurrence and metastases do occur. Recurrence with nodal metastases has been pathologically confirmed $[4,5]$; thus, the setting of newly diagnosed AFH may not parallel disease recurrence, whereas more-aggressive AFH may have higher rates of nodal involvement. Given the rarity of AFH, it may be difficult to perform a large, prospective evaluation of the rate of malignancy in suspicious nodes on imaging. This may be facilitated by the creation of prospective databases such as has been performed for sarcomas [6].

We showed that patients with newly diagnosed AFH often exhibit suspicious nodal lesions on imaging that are histologically benign. Less-invasive management of suspicious nodes, such as image-guided biopsy, may be 
preferable to nodal resections, if this will help decrease the aggressiveness of surgery with curative intent for newly diagnosed AFH.

Acknowledgments We thank Narasimhan Agaram MBBS (Department of Pathology, Memorial Sloan Kettering Cancer Center, New York, NY, USA) for assistance with pathology slides.

\section{References}

1. Antonescu CR, Dal Cin P, Nafa K, Teot LA, Surti U, Fletcher $\mathrm{CD}$, Ladanyi M. EWSR1-CREB1 is the predominant gene fusion in angiomatoid fibrous histiocytoma. Genes Chromosomes Cancer. 2007;46:1051-1060.

2. Chen G, Folpe AL, Colby TV, Sittampalam K, Patey M, Chen MG, Chan JK. Angiomatoid fibrous histiocytoma: unusual sites and unusual morphology. Mod Pathol. 2011;24:1560-1570.

3. Costa MJ, Weiss SW. Angiomatoid malignant fibrous histiocytoma: a follow-up study of 108 cases with evaluation of possible histologic predictors of outcome. Am J Surg Pathol. 1990;14:1126-1132.

4. Enzinger FM. Angiomatoid malignant fibrous histiocytoma: a distinct fibrohistiocytic tumor of children and young adults simulating a vascular neoplasm. Cancer. 1979;44:2147-2157.

5. Fanburg-Smith JC, Miettinen M. Angiomatoid "malignant" fibrous histiocytoma: a clinicopathologic study of 158 cases and further exploration of the myoid phenotype. Hum Pathol. 1999;30:1336-1343.

6. Fong Y, Coit DG, Woodruff JM, Brennan MF. Lymph node metastasis from soft tissue sarcoma in adults: analysis of data from a prospective database of 1772 sarcoma patients. Ann Surg. 1993;217:72-77.
7. Kao YC, Lan J, Tai HC, Li CF, Liu KW, Tsai JW, Fang FM, Yu SC, Huang HY. Angiomatoid fibrous histiocytoma: clinicopathological and molecular characterisation with emphasis on variant histomorphology. J Clin Pathol. 2014;67:210-215.

8. Kayton ML, Delgado R, Busam K, Cody HS 3rd, Athanasian EA, Coit D, La Quaglia MP. Experience with 31 sentinel lymph node biopsies for sarcomas and carcinomas in pediatric patients. Cancer. 2008;112:2052-2059.

9. Makis W, Ciarallo A, Hickeson M, Derbekyan V. Angiomatoid fibrous histiocytoma: staging and evaluation of response to therapy with F-18 FDG PET/CT. Clin Nucl Med. 2011;36:376379.

10. Martinez SJ, Moreno CC, Vinson EN, Dodd LG, Brigman BE. Angiomatoid fibrous histiocytoma: novel MR imaging findings. Skeletal Radiol. 2016;45:661-670.

11. Rossi S, Szuhai K, Ijszenga M, Tanke HJ, Zanatta L, Sciot R, Fletcher CD, Dei Tos AP, Hogendoorn PC. EWSR1-CREB1 and EWSR1-ATF1 fusion genes in angiomatoid fibrous histiocytoma. Clin Cancer Res. 2007;13:7322-7328.

12. Shi H, Li H, Zhen T, Zhang F, Dong Y, Zhang W, Han A. Clinicopathological features of angiomatoid fibrous histiocytoma: a series of 21 cases with variant morphology. Int J Clin Exp Pathol. 2015;8:772-778.

13. Thway K. Angiomatoid fibrous histiocytoma: a review with recent genetic findings. Arch Pathol Lab Med. 2008;132:273277.

14. Thway K, Fisher C. Angiomatoid fibrous histiocytoma: the current status of pathology and genetics. Arch Pathol Lab Med. 2015;139:674-682.

15. Ulaner GA, Magnan H, Healey JH, Weber WA, Meyers PA. Is methylene diphosphonate bone scan necessary for initial staging of Ewing sarcoma if 18F-FDG PET/CT is performed? AJR Am J Roentgenol. 2014;202:859-867. 